\title{
Feasibility of using acceleration-derived jerk to quantify bimanual arm use
}

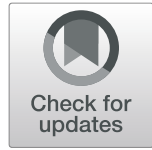

Ying-Chun (Preston) Pan ${ }^{1,2}$ E, Brianna Goodwin²,3 Emily Sabelhaus ${ }^{2}$, Keshia M. Peters ${ }^{3}$, Kristie F. Bjornson ${ }^{2,4}$, Kelly L. D. Pham ${ }^{2,5}$, William Walker ${ }^{2}$ and Katherine M. Steele ${ }^{3^{*}}$

\begin{abstract}
Background: Accelerometers have become common for evaluating the efficacy of rehabilitation for patients with neurologic disorders. For example, metrics like use ratio (UR) and magnitude ratio (MR) have been shown to differentiate movement patterns of children with cerebral palsy (CP) compared to typically-developing (TD) peers. However, these metrics are calculated from "activity counts" - a measure based on proprietary algorithms that approximate movement duration and intensity from raw accelerometer data. Algorithms used to calculate activity counts vary between devices, limiting comparisons of clinical and research results. The goal of this research was to develop complementary metrics based on raw accelerometer data to analyze arm movement after neurologic injury.
\end{abstract}

Method: We calculated jerk, the derivative of acceleration, to evaluate arm movement from accelerometer data. To complement current measures, we calculated jerk ratio (JR) as the relative jerk magnitude of the dominant (nonparetic) and non-dominant (paretic) arms. We evaluated the JR distribution between arms and calculated the 50th percentile of the JR distribution (JR50). To evaluate these metrics, we analyzed bimanual accelerometry data for five children with hemiplegic CP who underwent Constraint-Induced Movement Therapy (CIMT) and five typically developing (TD) children. We compared JR between the CP and TD cohorts, and to activity count metrics.

Results: The JR50 differentiated between the CP and TD cohorts (CP $=0.578 \pm 0.041$ before CIMT, TD $=0.506 \pm$ 0.026), demonstrating increased reliance on the dominant arm for the CP cohort. Jerk metrics also quantified changes in arm use during and after therapy (e.g., JR50 $=0.378 \pm 0.125$ during CIMT, $0.591 \pm 0.057$ after CIMT). The JR was strongly correlated with UR and MR $(r=-0.92,0.89)$ for the CP cohort. For the TD cohort, JR50 was repeatable across three data collection periods with an average similarity of $0.945 \pm 0.015$.

Conclusions: Acceleration-derived jerk captured differences in motion between TD and CP cohorts and correlated with activity count metrics. The code for calculating and plotting JR is open-source and available for others to use and build upon. By identifying device-independent metrics that can quantify arm movement in daily life, we hope to facilitate collaboration for rehabilitation research using wearable technologies.

Keywords: Rehabilitation, Cerebral palsy, Jerk, Activity count

\footnotetext{
* Correspondence: kmsteele@uw.edu

${ }^{3}$ Department of Mechanical Engineering, University of Washington, Seattle,

WA, USA

Full list of author information is available at the end of the article
}

(c) The Author(s). 2020 Open Access This article is distributed under the terms of the Creative Commons Attribution 4.0 International License (http://creativecommons.org/licenses/by/4.0/), which permits unrestricted use, distribution, and reproduction in any medium, provided you give appropriate credit to the original author(s) and the source, provide a link to the Creative Commons license, and indicate if changes were made. The Creative Commons Public Domain Dedication waiver (http://creativecommons.org/publicdomain/zero/1.0/) applies to the data made available in this article, unless otherwise stated. 


\section{Background}

Accelerometers, sensors that measure linear acceleration, have become a common tool to assess physical movement [1]. These small sensors provide objective and precise measures of motion and are ubiquitous in modern products such as smartphones. In addition to monitoring movement patterns of healthy adults [2], these sensors are also capable of quantifying motion in clinical populations [3-5]. Because of their portability, affordability, and continuous monitoring capability, accelerometers provide a quantitative adjunct to evaluating treatment efficacy and complement other in-clinic evaluations.

One clinical application of accelerometers has been to assess interventions to improve arm function after neurologic injury, such as among stroke survivors or individuals with cerebral palsy [6-12]. For these applications, accelerometer data have been used to analyze movement in terms of activity counts [13]. Activity counts have been shown to provide an accurate and repeatable measure of both the duration and intensity of activity, and have become one of the most commonly used measures for accelerometerbased rehabilitation research [14]. Accelerometers worn on each wrist have been used to calculate activity counts, which are then used to calculate common outcome measures that compare dominant and non-dominant arm use during clinical tests or daily activity. Some of the most common outcomes based on activity counts include the magnitude ratio (MR), which compares the relative intensity of movement between arms, and the use ratio (UR) which compares duration of arm use $[6,12]$. These metrics have been used to evaluate function before and after interventions and can detect clinically meaningful changes in function. For example, MR and UR have been used to evaluate movement at home and in the clinic for adult stroke $[15,16]$ and inform the efficacy of rehabilitation [17].

While pre-processed activity counts are convenient and demonstrate less variability than raw acceleration data, they are not standardized and depend on proprietary algorithms [13]. As highlighted by Hayward and colleagues (2010), "Different brands of accelerometers have different processes for integrating the signal to produce activity counts, which are not publicly available. This inherently makes it difficult to directly compare activity counts provided by different accelerometer brands" [18]. Even for devices from the same manufacturer, significant differences in activity counts have been reported, suggesting changes in algorithms that limit longitudinal evaluations [19]. This lack of transparency not only limits the understanding of the factors that influence these outcome measures, but also hinders clinicians' and researchers' ability to compare measures and interpret outcomes. While commercial platforms have decreased the cost of accelerometer technology, increased access, and created user-friendly interfaces, there remains a need to develop open-source algorithms that can capture clinically relevant changes in arm function. Ideally, such algorithms could use raw accelerometer data from any device to compare across studies.

The objectives of this research were to (1) derive a metric from raw accelerometer data to quantify arm movement, (2) evaluate whether this metric can quantify bimanual arm use for a rehabilitation intervention, and (3) provide open-source code for others to use and build upon. Based upon the early research and documented limitations of evaluating movement from raw acceleration data, we focused this analysis on jerk, the time rate of change in acceleration. As early as 1985, Flash \& Hogan described the coordination of arm movements with jerk, noting its advantage of capturing smoothness of movement [20], which is also commonly altered after neurologic injury [21]. Lucena and colleagues in 2017 showed the potential benefits of using jerk measured from inertial measurement units (IMUs) to evaluate bimanual arm use among stroke survivors [22]. They showed strong correlation with activity count measures; however, an IMU requires significantly more power, data storage, and cost than an accelerometer alone. In this research, we propose that jerk ratio (JR), a measure of the relative jerk between arms from wrist-worn accelerometers, is comparable to activity count measures in its ability to differentiate between children with unilateral cerebral palsy (CP) and typically-developing (TD) peers.

\section{Methods}

To evaluate jerk and activity count measures, we analyzed previously collected and reported data from five children with unilateral $\mathrm{CP}(3 \mathrm{M} / 2 \mathrm{~F}$, age: $7.2 \pm 0.5$ years, height: $125.5 \pm 9.3 \mathrm{~cm}$, weight: $29.0 \pm 11.7 \mathrm{~kg}$, average \pm one standard deviation) before and after CIMT and 5 TD peers (1 $\mathrm{M} / 4 \mathrm{~F}$, age: $7.8 \pm 1.1$ years, height: $127.6 \pm 8.3 \mathrm{~cm}$, weight: $25.8 \pm 1.8 \mathrm{~kg}$ ) [23]. While the original study had seven children in each cohort, we excluded patients who used a walker $(n=1)$ or who were outside of the age range between seven and nine to limit age effects $(n=1)$. The function of the children with CP were classified as Gross Motor Functional Classification System (GMFCS) Levels I-III, Manual Ability Classification System (MACS) Levels II-III, and had Functional Independence Measure for children (WeeFIM) self-care scores ranging from 2 to 7 (Table 1) [24-26]. CP03 had mild dystonia.

Accelerometers were placed on both wrists of the participants. Each child wore the sensors for three days during three separate time periods. The children with CP wore the sensors: 1) 1 to 2 weeks before the start of CIMT, 2) during the second week of CIMT, and 3) 6 to 8 weeks after CIMT, while the TD cohort wore the sensors during time periods temporally spaced to align with the data collections for the CP cohort. The CIMT protocol involved wearing a cast on the paretic arm for 3 weeks, with $2 \mathrm{~h}$ of occupational therapy focused on upper 
Table 1 Demographics and functional scales for the cerebral palsy group

\begin{tabular}{lccccc}
\hline & Gender & Age & GMFCS & MACS & WeeFIM $^{a}$ \\
\hline CP01 & M & 7 & $\|$ & $\|$ & 36 \\
CP02 & F & 7 & $\|$ & $\|$ & 35 \\
CP03 & M & 7 & $\|$ & $\|$ & 23 \\
CP04 & F & 8 & $\|$ & $\|$ & 30 \\
CP05 & M & 7 & । & $\|$ & 32 \\
\hline
\end{tabular}

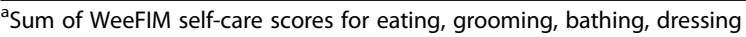
(upper and lower extremity), and toileting ( $6=$ dependence, and $42=$ complete independence)

extremity function at a tertiary children's hospital on weekdays during the treatment period. Previous research using activity count metrics from this dataset demonstrated significant differences between TD and CP cohorts, and significant changes in arm function during CIMT for the children with CP. Data were collected using the ActiGraph GT9X Link accelerometer (Actigraph, Pensacola, FL) at $100 \mathrm{~Hz}$. This small and wireless tri-axial accelerometer has a dynamic range of \pm 8 gravitational units. We synchronized the start time of the accelerometers using the ActiLife 6 software.

Data were stored on the device and raw accelerometer data were downloaded to a local machine using ActiLife 6. We applied a fourth order Butterworth bandpass filter with cutoff frequencies at 0.25 and 2.5 $\mathrm{Hz}$ to our data as this filtering scheme was shown to align with human motion [27]. We also obtained activity counts calculated during one-second time epochs using ActiLife 6 as our comparison measurement. We calculated the magnitude ratio (MR) and use ratio (UR) from the activity counts. The MR is calculated by taking the natural logarithm of the ratio of the vector magnitude of activity counts from the non-dominant arm and dominant arm for each time epoch [12]. To avoid infinite values, the MR excludes time points when either arm has zero activity. The UR is calculated as the duration of activity in the non-dominant (paretic) arm over that in the dominant arm, where duration is defined as the number of time epochs with activity count magnitude greater than or equal to two [6]. For all the children with $\mathrm{CP}$, the paretic arm was the child's nondominant arm.

To mirror these common activity count measures, we quantified upper limb movement with two outcome measures derived from jerk: jerk ratio (JR) and jerk ratio-50\% (JR50). Jerk is the time rate of change of acceleration and can be discreetly estimated by taking the difference of two subsequent acceleration measurements in each direction and dividing by the change in time. JR is defined as the ratio of the magnitude of jerk of the non-dominant (ND) arm over the sum of the magnitude of ND and dominant (D) jerk:

$$
\text { jerk ratio }(J R)=\frac{\left|N D_{\text {jerk }}\right|}{\left|N D_{\text {jerk }}\right|+\left|D_{\text {jerk }}\right|}
$$

Time points where |NDjerk| and |Djerk| were both zero were excluded from the analysis, although this was rare since raw acceleration measurements in $g$ are less processed than activity counts. For a given time point, a JR close to 1 or 0 suggests mostly non-dominant or dominant arm movement, respectively. JR was calculated for the collection period and filtered using a fourth order, lowpass Butterworth filter with a cutoff frequency of $3 \mathrm{~Hz}$. The rationale behind this secondary filter was to minimize noise that arises from changes in orientation. Without the magnetometer to detect device orientation, the present study assumes that jerk derived from rotational motion would both be random and high frequency.

Once JR was calculated, a probability density function (PDF) was estimated by normalizing the histogram with respect to the whole three-day collection period such that the total probability of the distribution is equal to one. In comparing PDF across collection periods, all PDFs were normalized by their maximum values. To provide a summary metric of the JR distribution, JR50 was calculated as the cumulative probability from $J R=0$ to 0.5 , where values over 0.5 suggest more frequent dominant arm use. Importantly, JR and JR50 are inversely related. In the case of high dominant arm use, the entire JR distribution could lie to the left of JR $=0.5$ and result in a JR50 of $1.0(100 \%)$ while the JR of individual time points might all be less than 0.5 .

We quantified the consistency of JR50 by comparing JR distributions under different conditions and calculating Pearson's correlation coefficient $(r)$. Similar to JR, MR is also a distribution of time points and summarized with MR50. To compare whether there are advantages to using jerk versus acceleration, we applied the same methods (eq. 1) to the raw acceleration data to calculate an equivalent acceleration ratio (AR). We used linear regression to compare JR and AR to activity count metrics (UR and MR) for TD and CP participants, evaluating the fit with Pearson's correlation coefficient $(r)$.

\section{Results}

For TD children, the distribution of JR during daily life was symmetric and had a median value of JR $50=$ $0.506 \pm 0.026$ (Fig. 1a). Examining the distribution of JR provides insight into the relative amount of unimanual versus bimanual activity in daily life. For TD children, this distribution indicates that there is more bimanual arm use than unimanual motion in daily life. The distribution was moderately repeatable between days and similar between TD children. The average similarity of JR distribution between collection periods was $0.945 \pm$ 0.015 . The average similarity of the JR distribution between TD children was $0.860 \pm 0.137$. 


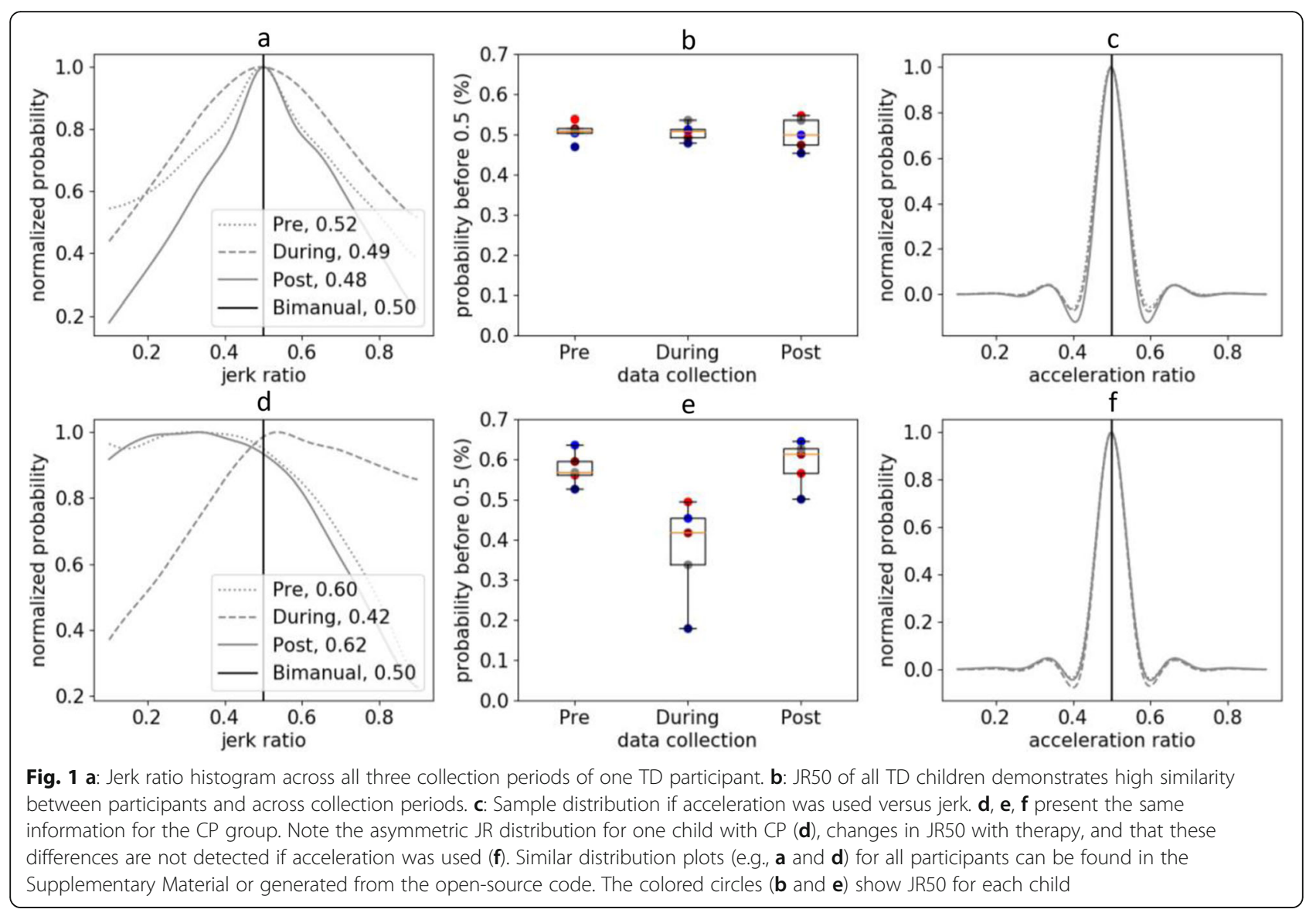

As expected, the distribution of JR before therapy was asymmetric for children with $\mathrm{CP}$ and had a higher median value of JR50 $=0.578 \pm 0.041$ (Fig. 1d), indicating that they were more reliant on their dominant, non-paretic arm in daily life before CIMT. The JR distribution was similar between the children with $\mathrm{CP}$, with an average similarity in JR distribution of $0.913 \pm 0.069$ before therapy. The JR was also sensitive to changes in arm use during therapy. The JR50 dropped during therapy to $0.378 \pm 0.125$, suggesting more paretic arm use during CIMT when the dominant arm was in a cast. However, the JR returned to baseline after CIMT with an average JR50 of $0.591 \pm 0.057$ (Fig. 2). The average similarity to the TD JR distribution was $0.726 \pm 0.184$ before therapy, $0.350 \pm 0.643$ during therapy, and $0.731 \pm 0.225$ after therapy. The changes in JR during treatment and compared to TD peers parallel the observations made from activity counts reported in the original analysis [23].

The JR was correlated with measures based on activity counts for both TD and CP cohorts (Fig. 3). In the CP cohort, JR correlated with UR and MR with an $r$ of -0.92 and 0.89 , respectively. In the TD cohort, JR correlated with UR and MR with an $r$ of -0.76 and 0.74 . We also evaluated whether similar conclusions could be drawn for ratios calculated from acceleration versus jerk.
In contrast to JR, we found that AR was unable to differentiate between the $\mathrm{CP}$ and TD cohorts and did not demonstrate changes during therapy (Fig. 1c, f). The AR was also poorly correlated with UR $(r=0.52$ for $\mathrm{CP}, r=-0.24$ for TD) and MR $(r=-0.68$ for $\mathrm{CP}$, $r=0.44$ for TD).

While the during-therapy JR distributions all shifted relative to pre- and post-therapy for all five $\mathrm{CP}$ participants, the shapes of these distributions showed differences between participants. For example, the duringtherapy curve for $\mathrm{CP} 05$ increased monotonically while CP02 had a convex distribution during therapy (Fig. 4). The monotonic increase in CP05 suggests that the participant could have been more cognizant about using their non-dominant arm to get the intended practice. Conversely, the convex shape in $\mathrm{CP} 02$ indicates that bimanual movement was still present, suggesting that the participant used their restrained arm during therapy.

\section{Discussion}

Calculating JR addresses limitations in contemporary accelerometer metrics by providing a sensitive metric of bimanual arm movement in daily life that avoids the use of activity counts derived from proprietary algorithms. Similar to activity count metrics (UR, MR), we found that the JR 


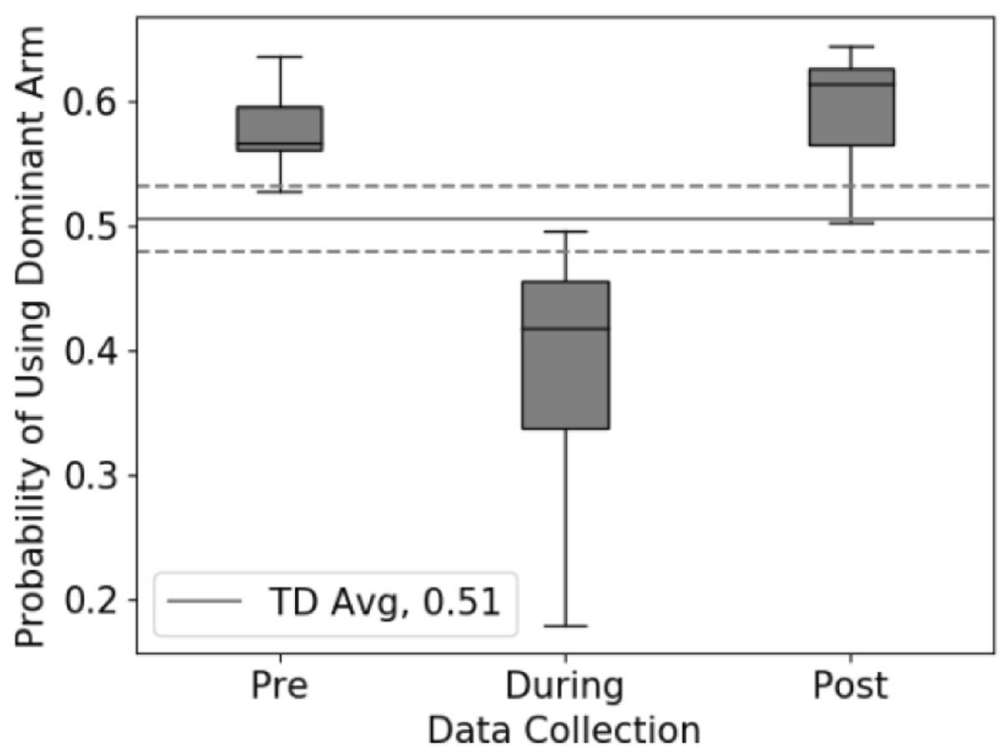

Fig. 2 The JR50 values of all CP participants demonstrate that before and after therapy, the children with CP are more reliant on their dominant arm than TD peers. During therapy, dominant arm movement is significantly reduced due to the cast. The dotted grey lines represent the range of JR50 values across the TD cohort

differentiated children with $\mathrm{CP}$ from TD peers and delineated changes in movement as children with $\mathrm{CP}$ participated in CIMT. Furthermore, the consistency of this metric across both collection periods and participants suggest good repeatability. There was also a strong correlation between JR and existing metrics; specifically, UR and MR calculated from the activity counts of one of the most commonly used commercial devices. While one could argue the JR lacks novelty as it resembles existing metrics, its potential lies in the cross-platform comparability as studies using different models of accelerometers could use JR to compare results using a shared, universal unit $(g)$. Furthermore, since the algorithm for this metric is open-source, others can build upon these findings in describing both the quantity and quality of arm motion.
Jerk has become an accepted kinematic metric in evaluating movement of individuals with neurologic disorders since the development of jerk-minimizing models of smoothness in 1985 [20]. Jerk-based metrics have been used with rehabilitation robots to measure smoothness of motion after stroke [28] and to analyze camerabased measures of movement in the home, such as with the Kinect depth camera [29]. While our research explored the potential of jerk to evaluate quantity of movement during daily life and clinical interventions, using jerk measured from wearable sensors to evaluate quality of movement represents an important area for future inquiry. In 2017, Lucena and colleagues derived jerk from a wrist-worn IMU and suggested "jerk asymmetry" and other metrics to understand the correlation between

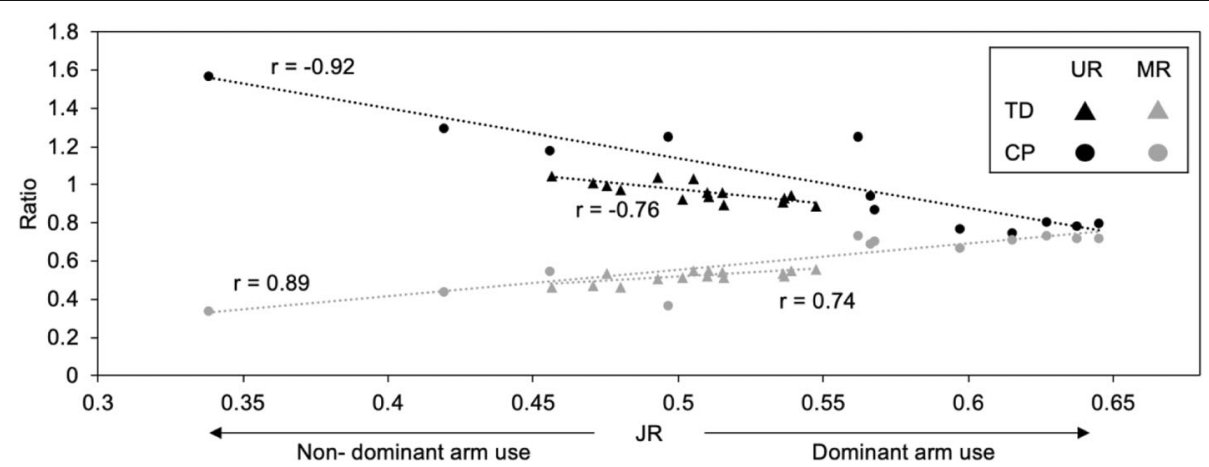

Fig. 3 Comparison of JR and activity count-based metrics. Note that as dominant arm use increases, UR decreases, while MR50 increases. Jerk ratio metrics were similar to activity count metrics for both the TD and CP cohorts 

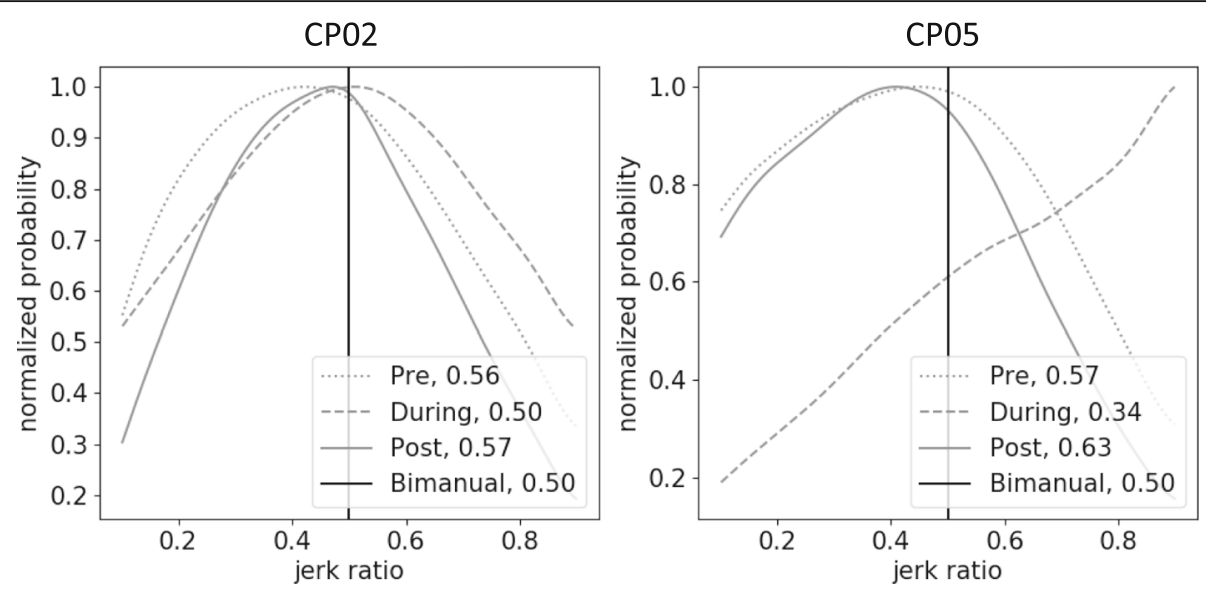

Fig. 4 Comparison of JR distributions between two CP participants. Note the difference in shape of distribution during therapy. This distinction might inform clinicians on the participants' activity level and alignment with therapy goals

kinematic metrics and existing functional tests. Using principal component analysis to identify the most indicative metric of human motion, they concluded that metrics based on acceleration and jerk contributed to the second principal component and accounted for $31 \%$ of variance across nine adult stroke survivors [22]. However, this work relied on an IMU, including both accelerometer and gyroscope, which increases sensor cost and decreases battery life. Our research builds upon this work and demonstrates that accelerometer-derived jerk is repeatable across data collections for TD children and can differentiate movement for a clinical population undergoing occupational therapy for the upper extremity. Our results show that accelerometers alone can produce similar measures to monitor bimanual arm movement in daily life.

Several limitations of the JR need to be considered. In order to capture the daily lives of participants, the collection period in this study was 3-days based upon prior research of individuals with $\mathrm{CP}$ [30]. However, due to variations in day-to-day activities, the peak of the JR distribution could vary between data collections. Since we were not focused on the absolute magnitude of JR, but rather the symmetry of the distribution and cumulative probability from JR $=0$ to 50 , we normalized each distribution by the maximum for comparison between days. Future research should investigate whether jerk magnitude should also be considered in the evaluation of movement intensity. The JR has limited ability in differentiating accelerations caused by rotational or linear movements because it does not use an IMU containing a gyroscope. However, by not focusing on any particular type of motion, this research supports using JR to quantify overall arm movement. While we were able to compare the results to clinical data, only a limited sample size was available for analysis ( $n=5$ in each cohort). The high similarity within the $\mathrm{CP}$ and TD cohorts suggests that JR demonstrates unique distributions in these populations that can be used for future comparison. However, future research will be required to investigate the JR distribution of other populations, such as adult stroke survivors or amputees.

To facilitate collaboration among researchers and encourage further development, the algorithm for calculating JR, as well as user-friendly code to produce plots similar to Fig. 1 are provided open-source as Python 3.6 code (Supplementary Material URL: https://steelelab.me. uw.edu/2020/02/jerk-ratio/). With this resource, research groups can use existing or newly created datasets from accelerometers to analyze $J R$ as a complementary metric to existing measures, enabling comparison between research studies or centers that may rely on different sensors and activity count algorithms.

\section{Conclusion}

The JR derived from raw acceleration data captured differences in motion between TD and CP cohorts and across different collection periods before, during, and after therapy. We found that JR correlated with existing activity count metrics including UR and MR that rely on proprietary algorithms. JR was repeatable between data collections for the TD cohort and exhibited high inter-subject similarity within both the TD and $\mathrm{CP}$ cohorts. The code for calculating and plotting jerk ratio is open-source and available in the Supplementary Material. By identifying device-independent metrics that can quantify arm movement in daily life, we hope to facilitate collaboration for rehabilitation research using wearable technologies. 


\section{Abbreviations}

AR: Acceleration ratio; CIMT: Constraint-Induced movement therapy; CP: Cerebral palsy; D: Dominant; IMU: Inertial measurement units; JR: Jerk ratio; MR: Magnitude ratio; ND: Non-dominant; TD: Typically-developing; UR: Use ratio

\section{Acknowledgements}

The authors would like to thank all participants, the occupational therapists, and the CIMT team at Seattle Children's Hospital.

\section{Authors' contributions}

PP collected parts of the data, developed the metrics, and drafted this manuscript. ES led the CIMT research project and secured funding. BG collected most of the data and provided feedback on the proposed metrics. KP provided feedback throughout the development of the metrics and edited the manuscript. KS led the research, secured funding, and edited the manuscript. ES and WW provided feedback and editing to the protocol and manuscript. All authors read and approved the final manuscript.

\section{Funding}

This research was supported by Seattle Children's Hospital Academic Enrichment Fund (\# 24080057) and the National Institute of Biomedical Imaging and Bioengineering of the National Institutes of Health under award number R01EB021935.

\section{Availability of data and materials}

Access to the datasets is restricted to UW researchers and employees of SCH, but algorithms for jerk ratio are open-source.

Project name: Jerk Ratio.ipynb

Project home page: https://colab.research.google.com/drive/164

vnXjduxpuSPt6A0KtO7alSK1Dlwi1l

(Shortened one to be provided in proof)

Operation system: Platform independent

Programming language: Python

No license needed.

\section{Ethics approval and consent to participate}

The procedures were approved by the Institutional Review Board at Seattle Children's Hospital and complied with the principles of the declaration of Helsinki. Parents of all participants provided written informed consent. Participants under the age of seven gave verbal consent, and those over seven signed an assent form as well.

\section{Consent for publication}

Not applicable

\section{Competing interests}

The authors declare that they have no competing interests.

\section{Author details}

'Department of Bioengineering, University of Washington, Seattle, WA, USA ${ }^{2}$ Seattle Children's Hospital, Seattle, WA, USA. ${ }^{3}$ Department of Mechanical Engineering, University of Washington, Seattle, WA, USA. ${ }^{4}$ Department of Pediatrics, University of Washington, Seattle Children's Research Institute, Seattle, WA, USA. ${ }^{5}$ Department of Rehabilitation Medicine, University of Washington, Seattle, WA, USA.

\section{Received: 25 June 2019 Accepted: 30 January 2020} Published online: 16 March 2020

\section{References}

1. Godfrey A, Conway R, Meagher D, ÓLaighin G. Direct measurement of human movement by accelerometry. Med Eng Phys. 2008;30:1364-86.

2. Ryan CG, Grant PM, Tigbe WW, Granat MH. The validity and reliability of a novel activity monitor as a measure of walking. Br J Sports Med. 2006;40: 779-84.

3. Cauwenberghe EV, Gubbels J, Bourdeaudhuij ID, Cardon G. Feasibility and validity of accelerometer measurements to assess physical activity in toddlers. Int J Behav Nutr Phys Act. 2011;8:67.
4. Pruitt LA, Glynn NW, King AC, Guralnik JM, Aiken EK, Miller G, et al. Use of accelerometry to measure physical activity in older adults at risk for mobility disability. J Aging Phys Act. 2008;16:416.

5. Tamura T. Wearable accelerometer in clinical use. Conf Proc IEEE Eng Med Biol Soc. 2005;7:7165-6.

6. Bailey RR, Lang CE. Upper extremity activity in adults: referent values using accelerometry. J Rehabil Res Dev. 2014;50:1213-22.

7. Coker-Bolt P, Downey RJ, Connolly J, Hoover R, Shelton D, Seo NJ. Exploring the feasibility and use of acceleromters before, during, and after a campbased CIMT program for children with cerebral palsy. J Pediatr Rehabil Med. 2017;10:27-36.

8. Lang, CE, Waddell, KJ, Klaesner, JW, Bland, MD. A method for quantifying upper limb performance in daily life using accelerometers. JoVE. 2017;122: e55673.

9. Uswatte G, Miltner WH, Foo B, Varma M, Moran S, Taub E. Objective measurement of functional upper-extremity movement using accelerometer recordings transformed with a threshold filter. Stroke. 2000; 31:662-7.

10. Noorkõiv M, Rodgers $\mathrm{H}$, Price $\mathrm{Cl}$. Accelerometer measurement of upper extremity movement after stroke: a systematic review of clinical studies. J NeuroEng Rehabil. 2014;11:144.

11. Michielsen ME, Selles RW, Stam HJ, Ribbers GM, Bussmann JB. Quantifying nonuse in chronic stroke patients: a study into paretic, nonparetic, and bimanual upper-limb use in daily life. Arch Phys Med Rehabil. 2012;93:1975-81.

12. Bailey RR, Klaesner JW, Lang CE. An accelerometry-based methodology for assessment of real-world bilateral upper extremity activity. PLoS One. 2014;9(7).

13. Tryon WW, Williams R. Fully proportional actigraphy: a new instrument. Behav Res Methods Instrum Comput. 1996;28:392-403.

14. John D, Tyo B, Bassett DR. Comparison of four Actigraph accelerometers during walking and running. Med Sci Sports Exerc. 2010;42:368-74.

15. Urbin MA, Waddell KJ, Lang CE. Acceleration metrics are responsive to change in upper extremity function of stroke survivors. Arch Phys Med Rehabil. 2015;96:854-61.

16. Shim S, Kim H, Jung J. Comparison of upper extremity motor recovery of stroke patients with actual physical activity in their daily lives measured with accelerometers. J Phys Ther Sci. 2014;26:1009-11.

17. Luster EL, McDaniel T, Fakhri B, Davis J, Goldberg M, Bala S, et al. Vibrotactile cueing using wearable computers for overcoming learned non-use in chronic stroke. In: 7th International Conference on Pervasive Computing Technologies for Healthcare and Workshops. 2013;378-81.

18. Hayward KS, Eng JJ, Boyd LA, Lakhani B, Bernhardt J, Lang CE. Exploring the Role of Accelerometers in the Measurement of Real World Upper-Limb Use After Stroke; 2016.

19. Rothney MP, Apker GA, Song Y, Chen KY. Comparing the performance of three generations of ActiGraph accelerometers. J Appl Physiol. 2008;105: 1091-7.

20. Flash $\mathrm{T}, \mathrm{Hogan} \mathrm{N}$. The coordination of arm movements: an experimentally confirmed mathematical model. J Neurosci. 1985;5:1688-703.

21. Krebs HI, Hogan N, Aisen ML, Volpe BT. Robot-aided neurorehabilitation. IEEE Transact Rehabil Eng. 1998;6:75-87.

22. de Lucena DS, Stoller O, Rowe JB, Chan V, Reinkensmeyer DJ. Wearable sensing for rehabilitation after stroke: Bimanual jerk asymmetry encodes unique information about the variability of upper extremity recovery. In: 2017 International Conference on Rehabilitation Robotics (ICORR); 2017. p. $1603-8$.

23. Goodwin BM, Sabelhaus EK, Pan Y-C, Bjornson KF, Pham KLD, Walker WO, et al. Children with cerebral palsy return to baseline community arm movement after constraint induced movement therapy. Am J Occup Ther 2020;74:5 in press.

24. Morris C, Bartlett D. Gross motor function classification system: impact and utility. Dev Med Child Neurol. 2004;46:60-5.

25. Eliasson A-C, Krumlinde-Sundholm L, Rösblad B, Beckung E, Arner M, Öhrvall A-M, et al. The manual ability classification system (MACS) for children with cerebral palsy: scale development and evidence of validity and reliability. Dev Med Child Neurol. 2006;48:549-54.

26. Ottenbacher KJ, Msall ME, Lyon NR, Duffy LC, Granger CV, Braun S. Interrater agreement and stability of the functional independence measure for children (weefim ${ }^{\mathrm{TM}}$ ): use in children with developmental disabilities. Arch Phys Med Rehabil. 1997;78:1309-15. 
27. John D, Freedson P. ActiGraph and Actical physical activity monitors: a peek under the hood. Med Sci Sports Exerc. 2012;44:S86-9.

28. Rohrer B, Fasoli S, Krebs HI, Hughes R, Volpe B, Frontera WR, et al.

Movement smoothness changes during stroke recovery. J Neurosci. 2002;22: 8297-304.

29. Won-Seok K, Cho S, Baek D, Bang H, Paik N-J. Upper Extremity Functional Evaluation by Fugl-Meyer Assessment Scoring Using Depth-Sensing Camera in Hemiplegic Stroke Patients. PLoS One; San Francisco. 2016;1 1:e0158640.

30. Mitchell LE, Ziviani J, Boyd RN. Variability in measuring physical activity in children with cerebral palsy. Med Sci Sports Exerc. 2015;47:194-200.

\section{Publisher's Note}

Springer Nature remains neutral with regard to jurisdictional claims in published maps and institutional affiliations.

Ready to submit your research? Choose BMC and benefit from:

- fast, convenient online submission

- thorough peer review by experienced researchers in your field

- rapid publication on acceptance

- support for research data, including large and complex data types

- gold Open Access which fosters wider collaboration and increased citations

- maximum visibility for your research: over $100 \mathrm{M}$ website views per year

At BMC, research is always in progress.

Learn more biomedcentral.com/submissions 